\title{
Neutrophils and lung injury: getting it right
}

\author{
Thomas R. Martin \\ The Medical Research Service of the VA Puget Sound Medical Center, and the Division of Pulmonary and Critical Care Medicine, \\ Department of Medicine, University of Washington School of Medicine, Seattle, Washington, USA
}

J. Clin. Invest. 110:1603-1605 (2002). doi:10.1172/JCI200217302.

Acute lung injury (ALI) is common in critically ill patients, and there has been a great deal of interest in the cellular and molecular mechanisms linking mechanical ventilation with ALI. Although it is possible to ventilate patients with normal lungs for prolonged periods of time without causing lung injury, a landmark controlled randomized clinical trial in critically ill patients with ALI showed that mechanical ventilation with a lung protective strategy improves outcome as compared with conventional mechanical ventilation (1). This trial was based on a series of studies in animals and humans suggesting that mechanical ventilation could injure the lungs, as measured by a number of different parameters $(2,3)$. In a separate study of humans with ALI, a lung protective strategy reduced cellular and biochemical parameters of the inflammatory response in the lungs, suggesting a key link between the mode of mechanical ventilation and inflammation observed in the lungs (4). The consistent association between polymorphonuclear neutrophils (PMNs) and lung injury in humans and animal models, and the propensity of PMNs and their products to cause tissue injury in experimental systems, led to the conclusion that PMNs have an important

\footnotetext{
Address correspondence to: Thomas R. Martin, Pulmonary Research, 151L, VA Puget Sound Medical Center, 1660 South Columbian Way, Seattle, Washington 98108, USA. Phone: (206) 764-2219;

Fax: (206) 768-5289;

E-mail: trmartin@u.washington.edu.

Conflict of interest: The author has declared that no conflict of interest exists.

Nonstandard abbreviations used: acute lung injury (ALI); polymorphonuclear neutrophil (PMN); ventilator-induced lung injury (VILI).
}

causative role in ALI $(5,6)$. Neutrophil depletion is protective in many animal models of ALI, and blocking the major PMN chemoattractant, IL-8, protects rabbits from lung injury and death following severe acid aspiration $(\mathrm{pH} 1.5)$ (7). Yet the results of these studies leave some questions unanswered, because none of the models closely simulate the events that occur in the injured lungs of humans. Although direct studies in humans are very limited, PMNs migrate into normal human lungs in response to an intra-alveolar chemoattractant without causing injury, and without undergoing significant degranulation (8). In addition, studies using G-CSF to augment host defenses in humans with severe pneumonia and sepsis showed that increasing the circulating PMN count to high levels was not associated with worsening clinical manifestations of lung injury $(9,10)$. Similarly, studies in sheep using the bidirectional movement of alveolar and vascular tracers showed that large numbers of PMNs migrate into the lungs in response to bacterial lipopolysaccharide without injuring the critical epithelial barrier of the lungs, even though endothelial permeability is increased (11). Careful ultrastructural studies in rabbits and mice have shown that PMNs migrate through specialized channels in endothelial and epithelial basement membranes and into the alveolar spaces in areas of streptococcal pneumonia without causing apparent damage to these barriers (12). Taking a different approach, a study of the metabolic activity of lung PMNs in rabbits with streptococcal pneumonia showed that the most metabolically active PMNs are in the alveolar spaces, and not in the microvasculature or the intersti- tium, suggesting that the major activation events occur in the alveolar spaces in response to bacteria, and not during migration (13).

\section{A link between PMNs and ventilator-induced lung injury}

Recognizing that none of the existing models are a perfect match for human disease, the resolution of these discrepant observations will require careful studies in experimental systems in which single variables can be manipulated. In this issue of the JCI, Belperio and colleagues take a molecular approach to studying the role of PMNs in ventilator-induced lung injury (VILI), using a scientifically straightforward but technically challenging model of VILI in mice mechanically ventilated with either small or large tidal volumes (14). The small tidal volumes and high respiratory rates of mice necessitate careful monitoring of systemic hemodynamics in order to ensure that the ventilatory protocols do not adversely affect hemodynamic parameters. An important consideration is that acute inflammatory mechanisms in mice differ in important ways from those of humans. For example, human PMNs express two major $\alpha$-chemokine receptors, CXCR1 and CXCR2, providing overlapping effector pathways for chemotaxis, whereas murine PMNs express only CXCR 2 , and lack CXCR 1 , which is the dominant chemotactic receptor for IL-8 on human PMNs. In addition, the inducible nitric oxide pathway is much more active in murine than in human macrophages. Belperio et al. exploited the lack of murine CXCR1 and the availability of CXCR2 knockout mice to test the role of PMN migration mediated by CXCR2 and its related ligands in the 


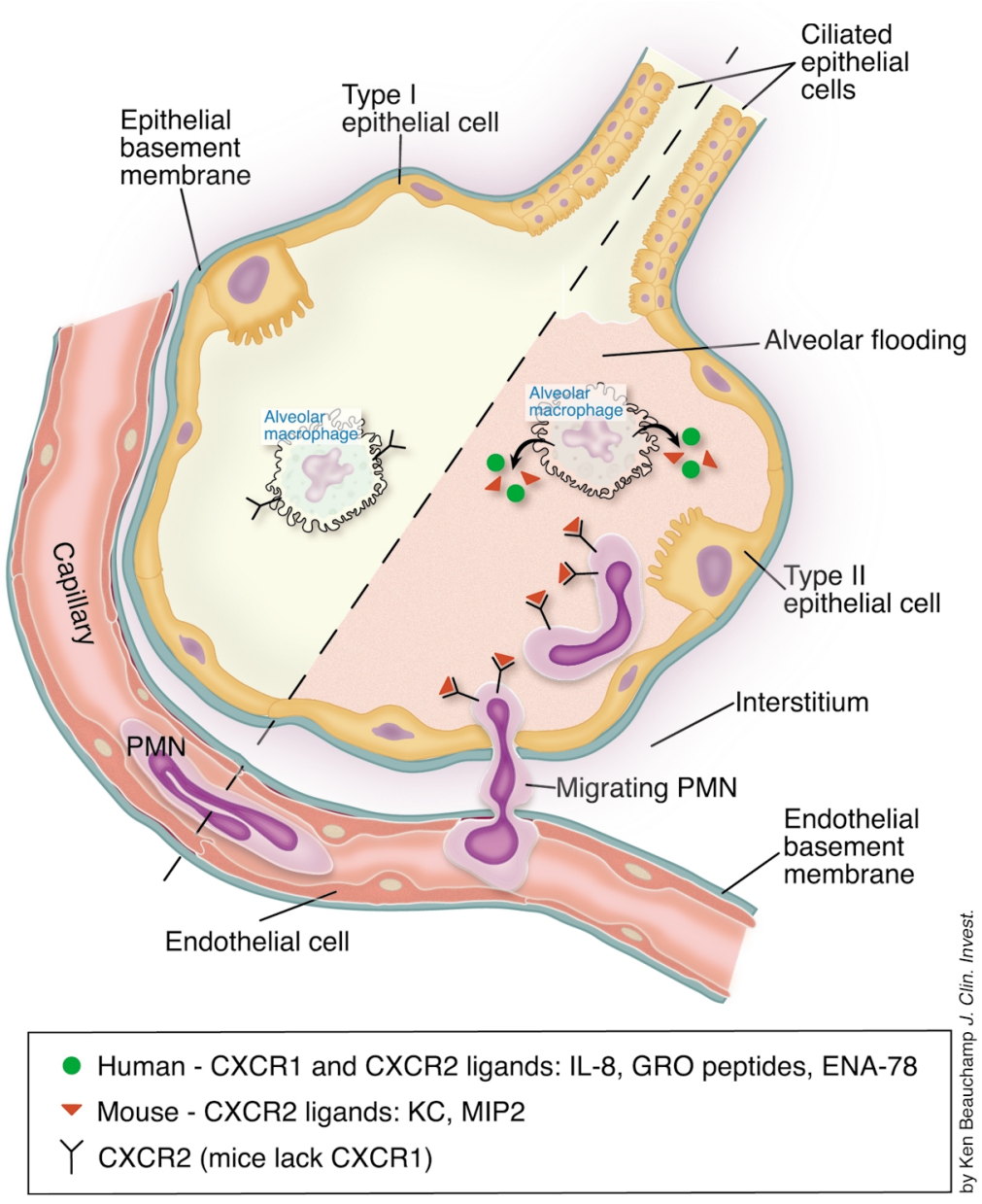

Figure 1

The normal alveolus (left side) and polymorphonuclear neutrophil (PMN) migration into alveolar spaces during a murine model of ventilator-induced lung injury (right side). Shown are the two critical barriers, the endothelium and epithelium. PMNs adhere to the capillary endothelium and migrate through the interstitium and the epithelium into the fluid-filled alveolar airspace. Alveolar macrophages secrete cytokines and interleukins that activate local neutrophils. In humans there are two major CXC receptors with overlapping ligands (CXCR1/IL-8; CXCR2/IL-8, GRO peptides, ENA-78). Mice have only CXCR2 (CXCR2/KC, MIP-2). The ligands are produced by alveolar macrophages and other cells in the lungs.

murine VILI model (14). Over a 6 hour period, the higher tidal volume strategy caused an increase in lung microvascular permeability, indicated by an increase in the extravasation of Evans blue dye into lung tissue, and an increase in the lung wet:dry ratio, which reflects extravascular lung water (Figure 1). Light microscopy showed alveolar flooding, PMN infiltration, and areas of alveolar hemorrhage. The myeloperoxidase content increased in lung homogenates suggesting PMN entrapment, and CXCR2 expression increased on leukocytes and other cells in the lungs. These findings were associated with an increase in CXCR2 ligands in lung tissue, and evidence of $\mathrm{I} \kappa \mathrm{B}$ phosphorylation and degradation,
PMNs. But do we have it right yet? The endpoints used by Belperio et al. are consistent with a change in microvascular permeability, whereas alveolar epithelial injury is the critical event that distinguishes ALI from lung edema due to a severe increase in hydrostatic pressure. In humans, ALI is associated with alveolar fibrosis and long-term disability, whereas successfully treated hydrostatic edema is associated with recovery of normal lung function. The study by Belperio et al. does not provide direct evidence about the status of the alveolar epithelium in the model, or the mechanism(s) by which it might be injured (14). As the authors note, their own light microscopy data suggest that epithelial damage occurred, but the level of resolution is not sufficient to prove this point. Prior studies have found ultrastructural evidence of epithelial damage in rats ventilated with very large tidal volumes, and that ventilating rats with lower tidal volumes reduces epithelial injury (15-17). Separation of endothelial and epithelial damage requires the use of bidirectional tracers to measure differential protein movement across the epithelial and endothelial barriers, careful ultrastructural studies, or the use of specific endothelial and epithelial markers $(11,17)$. The intriguing observation that CXCR2 expression increased on other populations of lung cells opens the possibility that CXCR2 has other important effects that need to be explored.

Despite the continuing need for more definitive studies about the fate of the alveolar epithelium in models of lung injury, the careful experimental approach taken by Belperio et al. leaves little doubt that PMNs are involved in the pathogenesis of lung microvascular injury associated with mechanical ventilation in this model, and that this is mediated by CXCR2 and its related ligands. Identifying the critical mechanism(s) by which higher stretch initiates this process, and the implications for epithelial injury, remain important experimental goals. The results of Belperio et al. suggest that by using similar molecular methods and additional experimental endpoints, we will eventually reach a more complete understanding of the role of PMNs in mediating epithelial as well as endothelial damage in ventilator-induced lung injury. 


\section{Acknowledgments}

This work was supported in part by the Medical Research Service of the Department of Veterans Affairs, and Grants HL65892, HL30542, and GM37696 from the National Institutes of Health.

1. NIH ARDSNet Group. 2000. Ventilation with lower tidal volumes as compared with traditional tidal volumes for acute lung injury and the acute respiratory distress syndrome. The Acute Respiratory Distress Syndrome Network. N. Engl. J. Med. 342:1301-1308.

2. Dreyfuss, D., and Saumon, G. 1998. Ventilatorinduced lung injury: lessons from experimental studies. Am. J. Respir. Crit. Care Med. 157:294-323.

3. Dos Santos, C.C., and Slutsky, A.S. 2000. Mechanisms of ventilator-induced lung injury: a perspective. J. Appl. Physiol. 89:1645-1655.

4. Ranieri, V.M., et al. 1999. Effect of mechanical ventilation on inflammatory mediators in patients with acute respiratory distress syndrome: a randomized controlled trial. JAMA. 282:54-61.

5. Repine, J.E., and Beehler, C.J. 1991. Neutrophils and the adult respiratory distress syndrome: two interlocking perspectives. Am. Rev. Respir. Dis. 144:251-252.

6. Lee, W.L., and Downey, G.P. 2001. Neutrophil activation and acute lung injury. Curr. Opin. Crit. Care 7:1-7

7. Folkesson, H.G., Matthay, M.A., Hebert, C.A., and Broaddus, V.C. 1995. Acid aspiration-induced lung injury in rabbits is mediated by interleukin-8 dependent mechanisms. J. Clin. Invest. 96:107-116

8. Martin, T.R., Pistorese, B.P., Chi, E.Y., Goodman, R.B., and Matthay, M.A. 1989. Effects of leukotriene $\mathrm{B}_{4}$ in the human lung. Recruitment of neutrophils into the alveolar spaces without a change in protein permeability. J. Clin. Invest. 84:1609-1619.

9. Nelson, S., et al. 1998. A randomized controlled trial of filgrastim as an adjunct to antibiotics for treatment of hospitalized patients with community-acquired pneumonia. CAP Study Group. J. Infect. Dis. 178:1075-1080.

10. Wunderink, R., et al. 2001. Filgrastim in patients with pneumonia and severe sepsis or septic shock. Chest. 119:523-529.

11. Wiener-Kronish, J.P., Albertine, K.H., and Matthay, M.A. 1991. Differential responses of the endothelial and epithelial barriers of the lung in sheep to Escherichia coli endotoxin. J. Clin. Invest. 88:864-875.

12. Walker, D.C., Behzad, A.R., and Chu, F. 1995. Neutrophil migration through preexisting holes in the basal laminae of alveolar capillaries and epithelium during streptococcal pneumonia. Microvasc. Res. 50:397-416.

13. Jones, H.A., et al. 1994. In vivo measurement of neutrophil activity in experimental lung inflammation. Am. J. Respir. Crit. Care Med. 149:1635-1639.

14. Belperio, J.A., et al. 2002. Critical role for CXCR2 and CXCR2 ligands during the pathogenesis of ventilator-induced lung injury. J. Clin. Invest. 110:1703-1716. doi:10.1172/JCI200215849.

15. Dreyfuss, D., Basset, G., Soler, P., and Saumon, G. 1985. Intermittent positive-pressure hyperventilation with high inflation pressures produces pulmonary microvascular injury in rats. Am. Rev Respir. Dis. 132:880-884.

16. Dreyfuss, D., Soler, P., Basset, G., and Saumon, G. 1988. High inflation pressure pulmonary edema Respective effects of high airway pressure, high tidal volume, and positive end-expiratory pressure. Am. Rev. Respir. Dis. 137:1159-1164.

17. Frank, J.A., et al. 2002. Low tidal volume reduces epithelial and endothelial injury in acid-injured rat lungs. Am. J. Respir. Crit. Care Med. 165:242-249. 